\title{
LA SPECULATION TRAVESTIE
}

\author{
GERARD LEBRUN \\ «Pour l'Allemagne, la critique de la religion \\ est faite en substance» \\ K. MARX. 1844.
}

L'affaiblissement et la suppression de l'individu est "le dernier écho du christianisme" dans la morale, lisons-nous dans l'aphorisme n. ${ }^{\circ} 132$ d'Aurore. Bien que l'abdication de l'ego, remarque Nietzsche, ne figure pas dans la doctrine du christianisme primitif, elle devint ensuite une des caractéristiques de la religion chrétienne. Et, de nos jours, positivisme, utilitarisme, socialisme, etc. vivent de cet héritage, et même renchérissent sur la passion chrétienne du nivellement. Dieu est mort, mais les hommes sont restés égaux et semblables, comme ils l'étaient sous son regard. Partis et sectes sont unanimes à "exiger que l'ego se nie lui-même" au bénéfice d'une communauté harmonieuse, "jusqu'à ce qu'il soit devenu quelque chose d'entièrement différent et nouveau".

Si nous passons de là à la critique anthropologique de Feuerbach dans l'Essence du Christianisme, nous trouvons exprimée l'opinion diamétralement opposée. La religion chrétienne selon Feuerbach est avant tout coupable d'avoir été centrée sur l'individu. D'où son infériorité, qu'il rappelle souvent, par rapport au paganisme, religion d'une communauté, religion du "genre":

'Les Anciens sacrifiaient l'individu au genre; les chrétiens sacrifient le genre à l'individu... Les chrétiens se distinguent des paiens en ce qu'ils identifient immédiatement l'individu avec le genre, et que chez eux l'individu a la signification du genre, l'individu pour lui-même étant tenu pour l'existence accomplie du genre" (1).

(1) Essence du Christianisme. trad. Osier (Maspero). p. 291, 293. 
Ce seul trait, en indiquant la différence d'inspiration des deux polémiques anti-chrétiennes, devrait nous mettre en défiance envers les rubriques dangereusement vagues de "démystification", de "pratique du soupçon", qu'on applique à la critique de la religion au $19 .^{\circ}$ Siècle dans son ensemble . Ces expressions recouvrent-elles les mêmes stratégies? Et n'est-il pas léger d'associer simplement les noms de Feuerbach, Marx et Nietzsche? Les textes qu'on vient de citer ébranlent au moins notre confiance en l'idée paresseuse d'une union sacréee des "démystificateurs". On rétorquera peut-être qu'ils ne concernent, après tout, qu'un point de fait très secondaire et que l'opposition sur ce point de Feuerbach et de Nietzsche n'implique pas encore que ceux-ci n'aient pas été, objectivement, les ouvriers de la même tâche.

Mais cette supposition est aventureuse. On s'en convaincra vite si l'on veut bien tenir compte des influences qui s'exercèrent sur Nietzsche et infléchirent sa critique religieuse - notamment celle de Max Stirner. Stirner, aujourd'hui, est surtout connu par les lourdes insultes dont il est gratifié dans "L'Idéologie allemande". Et c'est fâcheux. Car il est visible que Marx, pour les besoins de sa polémique, escamote ce qui est un des objectifs primordiaux de Stirner dans l'Unique et sa propriété: dénoncer en Feuerbach un néo-hégélien (2). Dans cet axe de lecture, l'oeuvre de Feuerbach apparaît plutôt comme une laïcisation de la dogmatique chrétienne, et toute la critique qu'il entreprend de celle-ci porte à faux. Ainsi, quel sens y a-t-il à reprocher au christianisme d'avoir glorifié "l'individu pour lui-même" et de l'avoir détaché au maximum de l'espèce? C'est le méfait inverse, répond Stirner, que commirent les chrétiens.

"C'est une illusion (de croire que) le christianisme accordait une valeur infinie au Moi... Non, il n'attribue cette valeur qu'à l'homme. Seul l'homme est immortel, et c'est seulement parce que Je suis homme que Je suis immortel. En fait, le christianisme a dû enseigner que personne ne disparaît, de la même manière que le libéralisme

(2) Sur la question du rapport de Marx à Stirner, nous renvoyons au très éclairant article de M. Henri Arvon: Une polémique inconnue, Marx et Stirner in Temps modernes, n. 71 septembre 1951. 
fait de tous des êtres égaux en tant qu'hommes; mais cette éternité et cette égalité ne concernaient que l'homme en Moi, et non pas Moi" (3).

Le christianisme au service de "l'individu", si l'on veut... Mais à condition de préciser: de l'individu humanisé, identifié à son être générique, échantillon standard du "troupeau". C'est en ce point que Stirner annonce Nietzsche, et, sans doute, l'inspire en partie. C'est à ce point aussi qu'il commence à démasquer en Feuerbach le hégélien mal repenti (4). D'où vient en effet cet opprobre jeté sur "l'individu pour lui-même" par Feuerbach et les feuerbachiens ${ }^{(5)}$ ? Il remonte, au moins, aux écrits anthropologiques de Kant: détaché du genre, l'individu n'est plus que sujet "pathologique", egoïste, rendu à l'animalité. "L'individu particulier est l'individu incomplet": ce principe pédagogique ouvre également la Phénoménologie, - et le thème est répercuté à travers tout le système hégélien, où le salut et la "libération" de l'individu sont toujours au prix d'une oblitération de sa différence, de sa transfiguration dans l'Universel (le divin ou l'Etat...). Rien de bien nouveau, donc, chez Feuerbach. Bruno Bauer, après Stirner, le constate: "Chez Feuerbach, l'individu doit se soumettre au genre et le servir. Chez Feuerbach, le genre est l'absolu hégélien". Que Feuerbach fasse consister la nocivité du christianisme dans l'attachement de celui-ci à "l'individu particulier" serait donc une preuve supplémentaire de son hégélianisme larvé.

On peut même aller plus loin. Ce lieu anti-chrétien lui-même, n'est-ce pas Hegel qui l'a fourni à Feuerbach? Un bon indice s'en trouve dans la lettre de novembre 1828 qu'envoie à Hegel le jeune Feeurbach, en même temps que sa dissertation d'habilitation. Il est douteux, certes, que le

(3) Stirner. L'Unique et sa propriété. trad. Gallissaire et Saugé (L'Age d'Homme). p. 225-226. - "Le christianisme, incapable de reconnaître l'individu comme unique, ne l'a conçu que dépendant; il ne fut en fait qu'une théorie sociale, une doctrine de la vie en commun, aussi bien de l'homme avec Dieu que des hommes entre eux. Aussi toute caractéristique individuelle propre - intérêt personnel, caprice, volonté personnelle, particularité, amour-propre... - devait-elle tomber avec lui dans le plus profund discrédit" (ibid. p. 221).

(4) C'est aussi la trèse que défend Bruno Pauer dans son article des "Norddeutsche Blätter" de 1844: "Ludwig Feuerbach", que prend à partie l' Idéologie allemande.

(5) Stirner, en citant La Question juive (p.226), range Marx parmi les feuerbachiens sectateurs de "l'authentique être-générique". 
maître ait beaucoup goûtê les thèses ultra-hégéliennes que défend ici le disciple. Mais il est symptômatique que Feuerbach ne craigne pas, dans cette lettre très respectueuse, de parler très naturellement du christianisme comme de l'erreur la plus tenace qui barre encore le chemin à la "réalisation" de la philosophie spéculative.

"Le christianisme ne peut être conçu comme la religion parfaite et absolue; celle-ci ne peut être que le royaume de la réalité de l'Idée et de la raison présente dans l'existence. Le christianisme n'est rien d'autre que la religion du Moi, de la personne en tant qu'elle est un seul esprit, et il n'est de ce fait que le contraire du monde antique... C'est pourquoi la raison n'est pas encore libérée dans le christianisme" ${ }^{(6)}$.

Feuerbach ne se souciait sans doute pas de scandaliser le "vénéré maître". Il faut donc croire que l'idée de la suppression effective de la religion ne lui semblait pas contraire à l'esprit de la philosophie spéculative, telle qu'il l'entendait à l'époque. La religion n'est-elle pas irréductiblement, de l'aveu même de Hegel, une figuration du divin à la mesure d'un esprit fini? De la réconciliation de l'essence divine et du Soi, la conscience chrétienne n'a jamais que la représentation. "Son apaisement reste lui-même affecté de l'opposition d'un au-delà. Sa propre réconciliation entre comme quelque chose de lointain dans sa conscience..." (7). La liaison entre Moi fini et religion chrétienne, c'est donc chez Hegel que Feuerbach la rencontre, - notamment dans les pages où Hegel montre comment le chrétien d'Eglise, tout en proclamant très haut son indignité, avoue par là même que son "moi fini" demeure son point fixe : "Cette humilité se contredit, elle est plutôt orgueil, car, si je pose le Vrai, c'est en l'excluant hors de moi, de sorte que je suis dans l'en-deçà, en tant que celui-ci (dieser), la seule instance affirmative, - de sorte que je suis l'étant en-soi et pour-soi par rapport auquel tout Autre disparaît. L'humilité véritable

(6) Feuerbach à Hegel. 22-II-28. in Hegel. Correspondance. III. trad. Carrère (Gallimard) p. 211-214.

(7) Phänomenologie (Glockner). II, S.601 - trad. Hyppolite. II, p. 289. Sur la "représentation" comme seule forme sous laquelle la conscience religieuse peut viser la Révélation, cf le paragraphe: "Rapport de la philosophie à la religion" dans l'Introduction des Leçons sur l'Histoire de la Philosophie (éd. Suhrkamp. XVIII, 81-113). 
renonce plutôt à soi, à ceci comme affirmatif" (8). Replié sur son egoïté, le chrétien est encore loin de cette humilité vraie.

Rien d'étonnant donc si, em 1828, Feuerbach, en tant que hégélien, tient pour incompatibles le "règne" du christianisme et celui de la philosophie nouvelle. Rien d'etonnant si, dès cette date, la mort du christianisme signifie l'abolition du "Moi fini", - de ce que Hegel appelle parfois ironiquement "le pieux individu" (9). Feuerbach, à ce moment-là, n'a conscience que de radicaliser l'analyse hégélienne. Ne peut-on dès lors présumer que, même après avoir reconnu en Hegel le restaurateur de la théologie chrétienne, il se fasse du "christianisme" un concept qui reste marqué par son apprentissage hégélien?

Toutefois, le rapport de Feuerbach à Hegel sur ce point est loin d'être aussi simple. Même s'il a retenu des thèmes de la philosophie hégélienne de la religion, Feuerbach n'a jamais repris l'analyse du christianisme que fait Hegel. Evitons toute équivoque. Par "analyse du christianisme", j'entends ici non pas la compréhension en dernière instance de ce que signifient les dogmes (il n'est que trop évident qu'il y a une différence entre la lecture spéculative et la lecture anthropologique), mais la compréhension de la façon dont le chrétien vit sa religion ou du sens littéral qu'ont les Ecritures ou les Pères de l'Eglise. La présentation de Feuerbach, ici, ne recoupe jamais celle de Hegel, tant dans les premiers écrits (de Berne et de Francfort) que dans l'oeuvre de maturité. Avant de tirer les conséquences de cela, je voudrais en donner rapidement quelques preuves.

En quoi consiste, dans l'Essence du Christianisme, le délire chrétien? A "unifier immédiatement" Dieu et cet homme-ci, - à donner au divin, c'est-à-dire au genre (Gattung) la forme de l'individu. Ou encore à proférer ces assertions fantastiques: le genre, c'est l'individu; l'illimité, c'est le limité ${ }^{(10)}$... A Autant le paganisme laissait entrevoir

(8) Ph. der Religion. XV, 199 (Glockner).

(9) “... Il est clair alors que le Moi (ainsi que les innombrables choses qui en dépendent cesse d'être considéré comme quelque chose d'absolument ferme, comme le principe général et déterminé du monde et de la représentation, que le Moi cesse d'être ce qu'il était jusqu'ici - bien plus, que le Moi expire" (lettre de Feuerbach à Hegel. p. 213).

(10) Cf Essence Chr. p. 447, et la présentation de M. Osier. p. 58-59. 
que "l'espéce humaine" était ce qu'adoraient les hommes sous le nom de "divin", autant le christianisme nous détourne de ce déchiffrement. Par là, il est la religion suprêmement mystificatrice, car il y a bien pire que de forger des dieux: il y a la croyance "superbe" que Dieu et moi ne faisons qu'un.

"Le chrétien, en tant qu'individu, n'est pas du même coup individu, mais genre, être universel, parce qu'il "possède la plénitude totale de sa perfection en Dieu", c'est-à-dire en lui-même (11).

Or, ce n'est certes pas cette mégalomanie que le jeune Hegel trouvait au coeur du christianisme. Ce qu'il ne lui pardonnait pas, c'était d'être une religion du déchirement, à l'inverse de la religion grecque qui savait unir en de "belles figures plastiques" la divinité des Olympiens et leur forme humaine. Le chrétien, tel que Hegel le représente à Francfort, éprouve sans doute le besoin d'une image de Dieu, mais en même temps l'impossibilité de jamais figurer l'infini, de viser tout à fait cet homme-ci (Jésus de Nazareth) comme Dieu ${ }^{(12)}$. - Chez Feuerbach, nous trouvons la thèse exactement inverse. Plus question d'une scission dans la conscience chrétienne entre Dieu et Jésus-Christ : le chrétien, au contraire, ne conçoit Dieu que sous la forme du Fils.

'Il est superficiel de dire que le christianisme n'est pas la religion d'un Dieu personnel, mais de trois personnalités... Seul le Christ est la personnalité plastique... Seul le Christ est le Dieu personnel. Il est le Dieu véritable et réel des chrétiens, ce qui ne peut être assez souvent répété' (13).

Si la conciliation du divin et du phénomène est le voeu de toute conscience religieuse, le christianisme est donc, "dans cette mesure", "la religion absolue, parfaite"... S'il en est ainsi, il pourrait sembler que Feuerbach reprenne en

(11) Ibid., p. 291.

(12) “Aussi est-il difficile de maintenir le côté religieux de Jésus ressuscité, l'Amour ayant pris forme en sa beauté; car c'est seulement dans une apothéose qu'il est devenu Dieu, sa divinité est une déification de quelque chose qt: est également donné comme réalité; il avait vécu comme individu humain, était mort sur la croix et avait été enseveli. Cette souillure de l'humanité est quelque chose de bien différent de la forme qui est appropriée au dieu... L'objectivité non divine, dont on exige aussi l'adoration, ne devient jamais du divin, malgré tout l'éclat dont elle rayonne" (Geist des Christentums. Suhrkamp. I, S. 409, 412). 
gros à son compte la compréhension du christianisme qui est celle du Hegel d'Iéna et de Berlin, même si c'est pour porter une appréciation toute différente. Certes, Hegel, dans la Philosophie de la Religion, est loin de présenter le christianisme comme une formation délirante, mais il comprend l'Incarnation comme l'union réussie de l'individu limité et du genre. C'est pourquoi le christianisme l'emporte alors pour lui de beaucoup sur l'anthropomorphisme grec qui ne faisait que déguiser les dieux en hommes: moins timide, il ose laisser se déployer le divin "jusqu'à l'extrême pointe de la réalité immédiate" (1t), - jusqu'à cet homme-ci. Pour la première fois, écrit-il, "Dieu est sujet et, en tant que subjectivité apparaissante, il n'est qu'un individu unique, exclusif" (15). Sommes-nous cette fois sur le terrain de Feuerbach, qui n'aurait fait, dès lors, que critiquer ce que louait Hegel dans l'Incarnation?

Tant s'en faut. A y mieux regarder, on s'aperçoit qu'il ne s'agit pas, ici et là, de la même Incarnation ni de la même religion. Quand Feuerbach parle du christianisme, il entend toujours et partout retrouver l'attitude de la conscience religieuse "finie" (pour parler le langage hégélien). Il ne sépare jamais le sens des dogmes et l'idéologie qui pourrait le déformer. Or cette distinction, chez Hegel, est capitale. Sans elle, on ne comprendrait pas son revirement par rapport au christianisme. Certes, bien des traits attestent l'attachement du chrétien à "l'être-fini", mais sa pratique, malgré l'apparence, est une abjuration de l'égoïsme. Et c'est à sa pratique qu'il faut le juger, non au déphasage qui lui interdit de penser pleinement ce qu'il vit. Ainsi, il est trop facile de railler dans la mentalité chrétienne une "liberté à l'égard des biens terrestres" qui ne va pas jusqu'à en abandonner la jouissance. Le christianisme, il est vrai, n'est pas une religion du sacrifice matériel; mais ce n'est pas "l'abandon d'une possession immédiate ou d'une existence naturelle", observe Hegel, qui

(14) Cf Vorlesungen weber die Gesch. der Philo. Suhrkamp. XIX, 508 sq.

(1.5) Ph. der Geschichte. XI, 417 (Glockner) - trad. Gibelin, P. 251. "(Chez les néo-platoniciens) l'Esprit n'est pas Esprit individuel; ce manque est comblé par le christianisme. Là l'Esprit est comme Esprit existant, présent, immédiat dans le monde; il est connu comme homme dans le présent immédiat, et tout individu possède pour soi une valeur infinie et une participation à cet Esprit, qui est né dans le cocur de tout homme. C'est ici que l'individu comme tel est libre, alors qu'en Orient un seul était libre, et quclques uns seulement chez les Grecs et les Romains. Dans le christianisme, an contraire, tout individu est la fin de la grâce de Dieu, et moi comme tel suis d'une valeur infinie" (Vorlesungen... XIX, 507). 
engage le plus la conscience religieuse ${ }^{(16)}$, - ce n'est pas la cession d'une récolte ou d'un troupeau. Moins dispendieux, mais plus décisif est le sacrifice symbolique par lequel je soumets à Dieu mon existence entière...

Compte tenu de cela, que signifie encore, pour Hegel, l'attachement du chrétien au fini? Même si le chrétien se pense naïvement comme une subjectivité subsistante face au Seigneur, il ne se comporte pas comme un possesseur indépendant face à un Maître plus puissant, mais de même race. Même s'il pense encore en "egoïste", il pressent pourtant - mieux qu'en toute autre religion - que la vie terrestre n'est que l'incessante négativisation de "l'être" dont la créditent païens et insensés; il ne vit le fini que pour se convaincre de sa nullité de tout instant. Bref, c'est la pratique néantisante du christianisme qu'il faut prendre en considération, non son idéologie "finitiste", - et l'interprétation hégélienne, alors, nous interdit de prendre pour une victoire de la subjectivité finie ce qui est au contraire la perpétuelle suppression de celle-ci, - l'avénement de la "subjectivité infinie". Chaque fois qu'il confesse que Dieu est son Soi vrai, le chrétien meurt en tant qu'individu naturel (17); il annule sa singularité finie bien plus radicalement que par l'ascèse ou le sacrifice. A partir de telles prémisses, il serait absurde de voir dans l'Incarnation une déification de l'individu, et Hegel ne manque pas de s'élever contre cette interprétation. Dans l'Incarnation, répète-t-il, l'important n'est pas l'haeccéité de Jésus en elle-même, la présence de cet individu-ci en ce lieu-ci. C'est pour l'avoir cru que le catholicisme laissa ses fidèles adorer les reliques et vivre dans la nostalgie du Ceci historique et terrestre, comme si la finitude n'avait pas été "seulement la forme" de

(16) Analyse du sacrifice dans $P h$. Religion. XV, 244. - Au contraire, sur l'incomplétude et l'hypocrisie du sacrifice, cf Systemfragment (1800) in in Frühe Schr. Suhrkamp. I, S. 424-425.

(17) C'est cette appropriation par le sujet de la vérité substantielle qui se réalise dans le lutherianisme. "Ainsi, ajoute Hegel, l'Esprit subjectif devient livre dans la vérité, il nie sa particularité et revient à soi-même en sa vérité" (Ph. Gesch. XI, 524 - tradl. p. 319). - C'est le moment où "le contenu de la vérité n'est plus détenu exclusivement par une caste", où la barrière s'abolit entre prêtres et läques Sur la signification de cette "Aufklärung" protestante, cf Stirner. Unique et sa propriété. p. 154-5: "(le luthérianisme) cherche à introduire l'esprit en toutes choses, à reconnaitre l'esprit saint comme leur essence, sanctifiant ainsi l' ensemble du temporel... Rien d'étonnant à ce que ce soit le luthérien Hegel - il explique quelque part qu'il "veut rester luthérien" - qui ait réussi cette pénétration totale du concept en toutes choses...". 
la Révélation (18). La venue du Christ, pour un luthérien, doit signifier tout autre chose: que le divin (l'Universel) s'atteste en subissant et en surmontant l'épreuve de la singularité absolue. L'admirable en l'Incarnation est que Dieu ne se soit pas perdu dans ce moment de l'unicité et qu'il ait fait se nier pour sa plus grande gloire jusqu'à la figue maudite de l'individualité (19). Entendue de la sorte, l'Incarnation chrétienne n'est nullement cette union magique et délirante du fini et de l'infini que décrira Feuerbach. Elle ne glorifie ni ne réhabilite le fini : elle l'exorcise.

'L'existence sensible où se trouve l'Esprit n'est qu'un moment passager. Le Christ est mort; c'est mort seulement qu'il est élevé au Ciel, assis à la droite de Dieu, c'est ainsi seulement qu'il est Esprit. Il dit lui-même : Quand je ne serai plus avec vous, l'Esprit vous conduira en toute vérité". Ce n'est qu'à la Pentecôte que les apôtres furent remplis de l'Esprit saint... Si le Christ ne doit être qu'un individu excellent, même sans péché et seulement cela, on nie la représentation de l'Idée spéculative, de la vérité absolue" (20).

Cette thèse d'une neutralisation de la finitude dans le christianisme, il est frappant que Feuerbach n'en tienne pas compte, même pour la réfuter. A quoi bon? Il est entendu que le chrétien est avant tout celui qui ne renonce qu'en paroles à une particularité qu'il se garde bien de sacrifier, et que sa religion consiste à "identifier immédiatement l'être particulier avec l'essence générale". Il est entendu que le christianisme est une divinisation de l'individu fini. Dès lors, les textes de Hegel relatifs à la "mort spirituelle" du chrétien ne méritent pas qu'on s'y arrête. Il va de soi encore que la "subjectivité infinie" chrétienne, telle que l'entend Hegel, est une expression dépourvue de sens. La "subjectivité" ne peut désigner que la centration sur soi du "pieux

(18) Ph. Religion. XV, 209-210 - Ph. Gesch. XI, 409, 480 - trad. p. 247, 292.

(19) La doctrine hégélienne semble être le commentaire de ce texte de Luther, cité par Feuerbach (in Essence Chr. p. 282): "La meilleure part de la résurrection est déjà arrivée; le Christ, chef de la chrétienté, est passé par la mort et ressuscité d'entre les morts. De plus, la partie excellente de moi-même, mon âme, est passée aussi par la mort, et est avec le Christ dans l'être céleste. En quoi donc peut me nuire le tombean, la mort?".

(20) Ph. Gesch. XI, 417 - trad. p. 251. 
individu", judaïque, puis chrétien (21), - et l'expression de "subjectivité infinie" est seulement caractéristique du procédé frauduleux qui consiste à proclamer l'union magique, chrétienne, du fini et de l'infini. - C'est là, bien sûr, un contresens et sur la philosophie hégélienne de la religion et sur la dialectique. Mais, pour Feuerbach, la dialectique n'est rien d'autre que cette fusion aberrante des incompatibles. Et cela mérite quelque réflexion.

Il est certain qu'on n'a pas de mal à entendre ainsi les propositions dialectiques: il suffit de les lire en maintenant la consistance et la fixité que l'Entendement donne à chaque terme. A ce compte, le persiflage est facile : Hegel en convenait le premier. Par l'opération du Saint-Esprit dialectique, dira-t-on, le fini est l'infini, le vivant singulier est le genre, le citoyen dans l'Etat est l'Universel... - Il me semble notable qu'aujourd'hui encore, cette lecture feuerbachienne, quand même sommaire, puisse être reprise sans l'ombre d'une réserve. Ainsi M. Osier, dans sa présentation de L'Essence du Christianisme, l'accepte, semble-t-il, sans nuances. Hegel, à l'en croire, affirmerait l'identité des contradictoires de la même façon que le prêtre catholique affirme que le pain est chair et que le vin est sang ${ }^{(22)}$. Enfin vint Feuerbach qui eut "beau jeu de rétorquer" que "ce n'est pas la dialectique hégélienne, mais le temps qui peut unir dans un même être les contraires et les contradictoires'... Ici, l'on s'étonne un peu: Feuerbach n'eut-il pas vraiment trop beau jeu, et suffisait-il de ce truisme pour faire s'effondrer la Logique de Hegel? Qu'on n'aille pas croire que je prenne la défense de Hegel. Je me demande seulement si une critique de Hegel est efficace, qui commence par l'interpréter en termes d'Entendement, c'est-à-dire contre son expresse volonté. Je ne soutiens nullement que le hégélianisme ne soit pas une pensée essentiellement chrétienne, mais je doute que la dialectique soit une transposition du délire religieux, un savoir immédiat à peine camouflé par du jargon. Pourquoi ne pas prendre plutôt Hegel à la lettre? Que la dialectique soit le discours de la mort au monde, que le christianisme ait été le système par excellence de la répression universaliste, n'y aurait-il pas là des chefs d'accusation assez graves pour Feuerbach et ses épigones? Il se peut que non...

(21) Sur l'égoïsme judéo-chrétien, cf Essence Chr. p. 465-466, - et les pertinentes remarques de M. Giannotti in Origines de la Dialectique $d u$ Travail, p. 60.

(22) Cf la présentation de M. Osier. p. 55-6. 
Quoi qu'il en soit, on ne saurait trop admirer la constance que met Feuerbach à faire dire à Hegel le contraire de ce qu'il disait ou, si l'on préfère, à dégager son "impensé".

J'en prends un exemple. La conscience religieuse, dit Feuerbach, sépare toujours à nouveau l'homme et Dieu pour en faire des existences indépendantes. C'est justement cette opposition d'Entendement, insurmontable pour le chrétien, que la spéculation hégélienne prétend abolir et dont le mouvement dialectique dénonce la vanité. L'étonnant est que Feuerbach ne mentionne même pas cet objectif de Hegel, et ajoute tout de go:

"La spéculation hégélienne identifie à présent ces deux côtés, mais de telle sorte pourtant que subsiste fondamentalement la vieille contradiction - elle est donc le développement conséquent, l'accomplissement d'une vérité religieuse. Dans sa haine contre Hegel, la foule érudite a été assez aveuglée pour ne pas reconnaître que sa doctrine ne contredit pas, du moins sous ce rapport, la religion - elle ne la contredit que de la manière dont en général la pensée cultivée et conséquente contredit l'imagination inculte et inconséquente qui pourtant énonce la même chose" (23).

La Philosophie de la Religion énonçant "la même chose" que la conscience malheureuse? Devant des affirmations aussi cavalières, faut-il parler de récusation de la dialectique ou d'imperméabilité à la dialectique? Certes, on n'ira pas soupçonner Feuerbach de mal connaître Hegel. On éprouve seulement, devant cette désinvolture, la même surprise qu'à la lecture de certaines polémiques d'Aristote où Platon est critiqué comme s'il n'avait jamais écrit le Parménide. Il est difficile de dire pourquoi un philosophe prend le droit de simplifier à l'excès et le risque de paraître malhonnête, ou quel enjeu est assez puissant pour lui imposer une lecture déformante. Dans le cas de Feuerbach, on l'entrevera peut-être tout à l'heure. - La tactique, en tout cas, est claire. Tout ce que le dialecticien décrit comme suppression idéale du fini, on l'entendra comme conservation sournoise du fini; quand le dialecticien prétend laisser se dire l'auto-supresion de la chose, c'est qu'il transfigue celle-ci pour la maintenir en place telle qu'elle est dans le fini. La pensée spéculative prend ainsi l'allure d'un monument d'hypocrisie.

(23) Essence Chr. p. 381. 
Veut-on voir cette méthode à l'oeuvre? La critique de la philosophie de l'Etat par le jeune Marx en est un bon exemple. Comment prendre Hegel au sérieux lorsqu'il nous représente l'entrée dans l'Etat comme l'une des formes de cette "mort" (très symbolique) de l'individu? L'anti-dialecticien a vite fait de déceler la supercherie: en réalité, "l'Etat moderne" hégélien est la sauvegarde de la propriété privée et de "l'individu libre" qu'elle a créé... Hegel, d'ailleurs, est bien près de l'avouer lorsqu'il oppose à "l'Etat moderne" la Cité substantielle antique dont la République de Platon fut la dernière image ${ }^{(24)}$. Platon entendait détruire tous les "germes' de la particularité (famille, propriété, libre choix de la profession). Le principe politique moderne, constate Hegel, est bien différent. C'est que le christianisme a éduqué la modernité; il a appris aux cités à s'accommoder de la subjectivité. Grâce à lui, nous savons que "le développement indépendant de la particularité" n'est plus forcément, comme le pensaient les Anciens, le début de la corruption et du déclin des cités. Nous savons que le maintien d'une sphère de la vie privée est non seulement compatible avec l'intégration de l'individu dans l'Etat, mais peut même constituer le meilleur ciment de celui-ci. - Revenons sur terre, répond le feuerbachien: loin que l'Etat politique moderne soit un mode d'intégration nouveau de l'individu, il est en realité la forme que se donne une société pathologique, "fondée sur des intérêts particuliers qui ne sont pas sociaux"; en réalité, l'Etat moderne ne peut être qu'un instrument d'oppression au service d'intérêts déterminés. Et la sophistique hégélienne, une fois de plus, a pour seul effet (sinon pour seul objectif) de sauvegarder et de normaliser la finitude, - en l'occurrence, cette coalition d'égoïsmes qu'est la société civile. Ici comme partout, la "suppression" dialectique de l'individuel dans son indépendance masque à peine la consolidation de cette indépendance. - Abstraitement (selon la lettre de Hegel), le Christ en tant qu'individu se supprime dans le divin; en réalité, la subjectivité finie demeure pour Hegel le réceptacle du divin. Abstraitement, la société civile trouve sa vérité/suppression dans l'Etat; en réalité, l'Etat est le prête-nom des électeurs censitaires. Telle est la règle du grand jeu de la "démystification": abstraitement, l'individuel se transfigure dans le genre; soyez attentif, et vous verrez qu'en réalité, le "genre" n'était que le masque de l'individuel, — et la spéculation un "empirisme

(24) Cf Ph. des Rechts. \& 185.

TRANS / FORM / AÇAO 1 49.70 
spéculatif" (25). Qu'on remplace "individuel" ou "finitude" par "amour de soi", et l'on comprendra pourquoi La Rochefoucauld est l'un des patrons de cette démystification.

Feuerbach n'a donc pas le même concept du christianisme que Hegel. Déification du fini, annulation du fini... Tout semblerait même en place pour un parallèle très scolaire. Car ce différend sur la signification d'une religion n'est qu'un point de fait, juste bon à éveiller une curiosité érudite. Plus intéressant est le parti-pris de Feuerbach qui le porte à lire Hegel comme si celui-ci avait en réalité repris et sauvegardé du christianisme cela même qu'à la lettre il en rejette. Prenons un dernier exemple. A suivre Feuerbach, la conception que se fait Hegel de l'Incarnation ne doit pas nous abuser: que le divin (l'essence humaine) ait pu se réaliser en cet homme-ci, Hegel, en réalité, l'admet au même titre que les catholiques. Sur ce point, spéculation et "représentation" religieuse sont d'accord. Il faut qu'elles le soient.

“...Est-il donc seulement possible que l'espèce se réalise de façon absolue dans un seul individu, l'art dans un seul artiste, la philosophie dans un seul philosophe... Sans doute, l'esprit, la conscience sont "l'espèce existant comme espèce"; mais l'individu, l'organe de l'esprit, la tête, aussi universelle soit-elle, sont toujours marqués, pointu ou camus, mince ou massif, long ou court, droit ou courbe, d'un nez déterminé... Rien ne se réalise sans se réaliser comme un être déterminé. L'espèce dans sa plénitude s'incarnant dans un individu unique serait un miracle absolu..." (26).

Ce "miracle absolu", Hegel ne se vante nullement de le réaliser. Il n'est que de se reporter aux textes: l'Incarnation n'a de sens que par le Golgotha, et la vie de cet homme-ci

(25) Feuerbach. Manifestes Philosophiques. trad. de M. Althusser (PUF). p. 28.

(26) Ibid. p. 14-15. - Dans cette page, Feuerbach parle du Messie comme du "Dalai Lama spéculatif". L'image, à coup sûr, vient de Hegel (cf Ph. Gesch. XI, 417 - trad. p. 451, et Ph. Religion. XVI, 311). Mais il est remarquable que Hegel oppose, au contraire, la singularité unique du Christ aux lamas qui sont les exemplaires multiples d'un Dieu qui demeure substance. 
était seulement nécessaire pour que Dieu s'accomplît jusqu'en cet extrême négatif qu'est l'existence d'un mortel. Mais Feuerbach, nous le savons, ne veut pas entendre ce langage. Hegel accepte que l'infini s'unisse à du déterminé, il consent à cette absurdité : Feuerbach ne sort pas de là .

Prêtons attention à cette étrange critique. On en pénétrerait mal le sens si l'on s'en tenait à l'image traditionnelle et très simplifiée qu'on se donne souvent de Feuerbach, apologiste de l'immédiat et du sensible, pourfendeur de l'abstraction. Ce n'est pas seulement cela qui est en question, lorsque Feuerbach s'attaque au christianisme et à Hegel en tant que chrétien. Ce n'est pas de cela qu'il s'agit exactement. Il y a deux points à distinguer dans la polémique: Hegel est $1 .^{\circ}$ ) un penseur abstrait, $2 .^{\circ}$ ) dans la ligne de l'idéologie chrétienne.

En tant que penseur abstrait, Hegel "prend presque toutes les choses dans un sens où on ne les reconnaît plus"; il utilise leur nom "sans pourtant conserver la valeur du concept qui est lié à ce nom" (27). Rien de plus juste: les mots sujet, Concept, etc. n'ont plus rien de commum (le lecteur de Hegel en est assez averti) avec leur signification populaire, "représentative". Cette mutation de sens, pense Feuerbach, est indissociable de l'aliénation propre à toute théologie; c'est en tant que théologien que Hegel, systématiquement, dépossède l'homme et le monde de leur essence. "La philosophie de Hegel a fait de la pensée, de l'être subjectif, mais pensé sans le sujet, et donc représenté comme un être distinct de lui, l'être divin et absolu (28). C'est ce thème-là qu'on a surtout retenu de la critique de Feuerbach. C'est un autre qui doit nous intéresser ici.

Si Hegel est chrétien, c'est plus précisément parce qu'il transfère (ou est censé transférer) dans le Dieu abstrait la divinité du Moi, - parce que son Dieu est tel que la conscience singulière rencontre en lui sa propre divinité. C'est la raison pour laquelle il est au plus loin de percer le secret anthropologique de la religion. Sans doute, l'anthropologie est inscrite à l'encre sympathique dans la "vraie théologie" hégélienne : c'est le thème du chapitre de l'Essence consacré à "la doctrine spéculative de Dieu". Sans doute il suffirait d'une très légère inflexion pour que le texte de Hegel avoue

(27) Manifestes. p. 163.

(28) Ibid., p. 161. 
la vérité du christianisme : on peut y apprendre que "c'est seulement dans l'homme que se réalise, se manifeste l'essence divine en tant que telle" (29). A ce point, il en faudrait donc très peu pour reconnaître que "la conscience humaine est conscience divine". "Pourquoi alors, s'écrie Feuerbach, aliéner à l'homme sa propre conscience, et en faire la conscience de soi d'un être séparé de lui, d'un objet?". Oui, pourquoi? Sinon parce que Hegel reste enfermé dans la vieille opposition théologique: divin/humain, essence infinie/Moi fini et qu'il est donc incapable de désigner "l'unité véritable, autarcique des essences divine et humaine" (30). Voilà en quoi Hegel est au plus loin d'entrevoir ce qu'il fait pourtant deviner à son lecteur. Lui aussi sépare en secret le fini et l'infini; à son tour, il donne au Moi singulier assez de consistance pour devoir s'interdire de verser la "divinité" au compte de l'être humain. - Feuerbach découvre en somme que Hegel est encore tributaire de cette "pensée finie" ou "pensée d'Entendement" qu'il avait dépistée à travers la tradition. En retournant contre Hegel lui-même l'accusation de "finitisme", sa critique est ainsi ultra-hégélienne.

Qu'en est-il alors de Feuerbach penseur de la finitude? Entendons-nous. Il est vrai que l'anthropologie rend au fini ce que l'abstraction en avait détaché : l'homme est la vérité de Dieu, le fini est la vérité de l'infini (31). Mais on aurait tort de croire que, par là, la notion abstraite d' "infini" est purement et simplement annulée. S'il en était ainsi, pourquoi Feuerbach tirerait-il gloire d'unifier une bonne fois, sans risque de fêlure, les essences divine et humaine? Bien sûr, le mot divin est alors à mettre entre guillemets, mais il reste qu'il serait absurde de parler d'unité et d'unification si le divin (religieux) n'avait jamais été qu'une sublimation fantastique. Cela, d'ailleurs, Feuerbach se défend de l'avoir suggéré :

"Si mon livre ne comportait que la seconde partie, on aurait sans doute absolument raison de lui reprocher une tendance exclusivement négative, et de voir dans la proposition : la religion est néant et non-sens, son contenu essentiel. Mais je ne dis nullement (et pourtant comme cele m'eût arrangé) : Dieu est néant, la Trinité est néant, la

(29) Essence Chr., p. 379.

(30) Ibid., p. 382.

(31) Manifestes. p. III. 
parole de Dieu est néant, etc. Je montre seulement qu'ils ne sont pas ce qu'ils sont dans l'illusion de la théologie..." (32).

L" "être infini" est donc plus qu'un "ens imaginarium" que ferait s'effacer le démystificateur, à mesure qu'il retrouve, sous les détermination abstraites, les déterminations finies dont provenaient celles-ci. Aussi ne suffit-il pas de retracer la genèse des propriétés divines à partir du fini, de reparcourir le chemin de "l'abstraction": il faut aussi dévoiler le sens véritable qu'avait l'infinité de Dieu. Il ne suffit pas de détronêr Dieu en tant que personnage fabuleux : il faut aussi déterminer en quel sens nous devons prendre sa "toute-suffisance", sa "toute-puissance", sa "suprême bonté"... “Il est infini au sens où le genre est infini, n'est pas limité à un lieu, un temps, un individu, une espèce... il est au-dessus des hommes au même sens où la couleur est au-dessus des couleurs, l'humanité au-dessus des hommes... il est l'être parfait au sens où le genre l'est par rapport aux individus..." (33). Autre chose est donc de prouver que le contenu du divin n'était en tous points que la vérité $d u$ fini, autre chose de pénétrer sa signification et de comprendre quelle est la véritable infinité, la véritable illimitation.

La suggestion de Stirner d'où l'on était parti prend alors toute sa portée: Feuerbach ne serait-il pas avant tout l'héritier de Hegel? En analysant le jugement que l'un et l'autre portent sur le christianisme, en établissant que le christianisme vu par Feuerbach n'a rien de commun avec le christianisme vu par Hegel, il pouvait sembler qu'on invalidait cette hypothèse. Nous voyons maintenant qu'il n'en était rien. C'est qu'il n'y avait pas d'opposition à proprement parler dans cette trame de Marivaux que l'on peut résumer ainsi : la preuve, pour Feuerbach, de l'allégeance de Hegel au christianisme est qu'il comprend le christianisme de la manière dont le Hegel historique assurait qu'il ne fallait pas le compreendre. Une telle "opposition" ne recouvre-t-elle pas plutôt une complicité dans les présupposés? Si je condamne dans le christianisme une glorification de l'individu, si vous le célébrez pour avoir été la religion de la désindividualisation, notre querelle herméneutique n'est-elle pas l'indice que nos présupposés philosophiques sont les mêmes? que ma critique et votre apologie s'inspirent du même idéal... Dès

(32) Essence Chr. p. 107.

(33) Ibid., p. 446-447. 
lors, rien de suprenant si le hégélianisme est en fin de compte déplacé plutôt que "renversé" et si l'infini théologico-spéculatif ne fait, très exactement, que changer de site.

'Le mystère de la plénitude inépuisable des déterminations divines n'est donc rien d'autre que le mystère de l'essence humaine, en tant qu'elle est infiniment variée, infiniment déterminable mais aussi, pour cette raison précisément en tant qu'être sensible. C'est seulement dans la sensibilité, dans l'espace et le temps, qu' a sa place un être infini, réellement infini, riche en déterminations" ${ }^{(34)}$.

Où est ici le "renversement"? Il s'agit plutôt de l'inversion d'un transfert :

"La tâche de la vraie philosophie est de reconnaître non pas le fini dans l'infini, mais au contraire le non-fini, l'infini dans le fini; en d'autres termes non pas de transposer le fini dans l'infini, mais de transposer l'infini das le fini" (35).

Ainsi, l'infini aura nom genre humain et non plus Esprit. Mais qu'apporte de nouveau ce changement dans la nomenclature? Les acteurs seuls ont changé, et non les rôles. Et n'est-se pas encore trop dire? Il semble qu'il y ait eu parfois simple modification dans la distribution plutôt que distribution nouvelle. L'infinité du "Geist" a fait place à celle de l'espèce. Mais déjà pour Hegel la puissance de l'espèce était une image naturelle de l'incomplétude de l'individu par rapport à l'universel. Le "naturalisme" consiste-t-il donc à prendre cette image pour vérité dernière? - On peut aussi bien s'interroger sur l'originalité de la thèse "humaniste". L'individu humain, affirme Feuerbach, est le seul être à vivre relativement à son espèce et à objectiver son genre. Là dessus, consultons le lexique hégélien : nous verrons que le "Geist" n'est rien d'autre que l'objectivation du genre.

"Cette différence (de la subjectivité singulière et du genre) est un processus qui a le résultat suivant : le genre vient à lui-même en tant qu'uni-

(34) Ibid., p. 140-141.

(35) Manifestes. p. 111. 
versel, et la singularité immédiate est niée. Cette disparition, c'est la mort de l'individu. La nature organique finit là où, à la mort de l'individu, le genre vient à lui-même et devient ainsi son propre objet, - ce qui est le surgissement de l'Esprit" (36).

Laissons une dernière fois parler les textes. - Quelle est, chez Feuerbach, la fonction du "genre humain" entendu comme infini réel? Montrer la fragilité des limitations individuelles, faire éclater systématiquement leurs bornes (ce dont se souviendra Marx dans son analyse de la spécificité du capitalisme) .

"Ma vie est liée à un temps limité, celle de l'humanité non. L'histoire de l'humanité n'est pas autre chose que la victoire continuelle remportée sur les limites qui à une époque déterminée étaient tenues pour les limites de l'humanité, et pour cette raison, pour des limites absolues, indépassables. Mais l'avenir dévoile toujours que les prétendues limites du genre n'étaient que celles des individus" (37).

Quel est le rôle du "Geist" hégélien? Etre le principe de transgression de toute limitation ${ }^{(38)}$. Là encore, renversement ou déplacement? Cette question, on peut d'ailleurs se la poser dès les premières pages de l'Essence, lorsque Feuerbach, définissant la religion comme la conscience de l'infinité de l'essence humaine, ne fait que reprendre et commenter le mot de Hegel: "Connaître sa limite, c'est connaître son illimitation". Début inattendu, on l'avouera, pour un livre dont on nous assure encore qu'il contient "la destruction

(36) System der Philosophie. \& 367. Zusatz. IX, S. 668 (Glockner).

(37) Essence Chr. P. 292.

(38) "La forme de l'Esprit est essentiellement celle de l'être-nié du fini. En lui, le fini a seulement la signification d'un être-supprimé et non d'un étant. La qualité propre de l'Esprit, c'est plutôt là véritable infinité, c'est à dire cette infinité que, au lieu de s'opposer unilatéralement ou fini, détient le fini en elle à titre de moment. Il est donc vain de clire qu'il y a des "esprits finis". L'Esprit en tant que tel n'est pas fini: il a la finitude en lui, mais seulement comme quelque close qui cloit être supprimé et qui est supprimé" (System. \& 386 Zusatz. X, 43). - Cf in Revue Int. Philo. n. ${ }^{\circ} 101$ l'article de M. Michel Henry: La critique de la religion et le concept de genre, où l'auteur conclut, preuves à l'appui, que l'anthropologic de Feuerbach est "une pâle réplique de l'ontologie hégélienne": "l'anthropologie... n'accomplit rien, ni la moindre mutation conceptuelle, ni le moindre changement dans le contenu des concepts qu'elle emprunte". 
totale" des présupposés hégéliens ${ }^{(39)}$. - Certes, il y a le témoignage d'Engels sur la rupture que marqua le livre de Feuerbach, le coup de tonnerre qu'il fut pour sa génération : "L'enchantement était rompu; le "système" (de Hegel) était brisé et jeté au rancart". Document intéressant pour l'historien des idées, mais thèse que rend intenable le moindre examen des homologies thématiques. Que Feuerbach ait libéré ses disciples de la dialectique, nul doute; du hégélianisme, c'est une autre affaire. Pour jeter le hégélianisme au rancart, il ne suffit tout de même pas de faire de la dialectique une variété de délire, de l'“Aufhebung" une imitation du miracle et de Hegel un incorrigible "idéaliste". Chaque fois que vous entendez parler doctement de "l'idéalisme hégélien", soyez sûr que le critique (ou le rabâcheur) n'a pas songé que bien des évaluations hégéliennes pourraient encore loger en son discours: l'expression "idéalisme hégélien" - c'est ainsi - est devenue le sûr indice de cette égayante ingénuité. De ces évaluations, je ne ferai pas ici le compte. Citons-en deux, seulement. Que le genre soit la destination de l'individu, que l'universel s'atteste dans l'effacement des limitations, voilà des convictions tenaces qui survivent au "renversement" - et, qui sait? se faufilent à travers les "coupures". - L'avertissement de Stirner est donc à prendre au sérieux: il se pourrait que l'"anthropologie", spéculation travestie, soit, par ce biais, "la dernière métamorphose de la religion chrétienne". Contre cette assertion, Feuerbach s'insurge. Mais que vaut sa défense?

Stirner, dit-il, pense que je sacrifie le "moi individuel et réel" à une abstraction: l'Homme. Etrange reproche, vraiment, à un livre qui est la première divinisation de l'individu humain.

"Unique, as-tu lu entièrement l'Essence $d u$ Christianisme? C'est impossible; car quel est jus-

(39) Cf la présentation de M. Osier. p. 59. - Ce qu'on peut se permettre de reprocher à $\mathrm{M}$. Osier, c'est de conclure à la destruction du hégélianisme, sans avoir ouvert le dossier du rappont Hegel - Feuerbach et en reprenant à son compte l'idée feuerbachienne de la dialectique, qui rend dès lors trop facile la critique de Hegel. - Il est vrai que M. Osier est marxiste, et, pour lui, le problème n'est pas là. Etant entendu que Feuerbach nous a libérés cle "l'incantation" hégélienne et cu'il nous a permis de "penser tout court", l'intérêt est de comprendre pourquoi "ce privilège n'était pas réservé à Feuerhach". Pour nous, nous demandons qu'on n'aille pas si vite et qu'on n'évite pas la question préalable: et si Feuerbach ne nous avait délivrés d'aucune incantation? 
tement le thème, le coeur de ce livre? Uniquement et purement la suppression de la scission en un moi essentiel et un moi inessentiel - la divinisation, c'est-à-dire la position, la reconnaissance de l'homme total, de l'homme de la tête aux pieds. La divinité de l'individu n'est-elle pas expriméee en toutes lettres dans la conclusion comme le mystère résolu de la religion?" (40).

En ces lignes, Feuerbach souligne le mot individu. Mais c'est le mot divinité qui retient l'attention de Stirner : cet "individu" divinisé me désigne-t-il encore, moi? le sens du mot n'est-il pas mystifié, à la manière hégélienne? - A partir de là, chacun des adversaires va renvoyer à l'autre l'accusation de crypto-christianisme. Certes, dit Fuerbach, je parle de l'individu, je ne parle même que de lui, mais non de "cet individu-ci", - ce qui serait "retomber dans le point de vue de la religion" (41), à l'exemple de Stirner, qui ne fait que transposer en son Unique le Dieu du monothéisme. Qu'il y ait divinisation de l'espèce humaine, et non de l'individu, atteste au contraire que l'émancipation du christianisme est complète.

"Ce point de vue, tu ne peux le supprimer qu'en transportant cet individu incomparable des nuées éthérées de son égoïsme surnaturel dans l'intuition sensible profane, qui fera ressortir, certes, sa particularité individuelle, mais du même coup aussi, d'une façon incontestable et indéniable, son identité et sa communauté avec les autres individus. Ne donne pas moins que son dû à l'individu singulier, mais aussi ne lui donne pas plus. C'est ainsi seulement que tu pourras te libérer des chaînes du christianisme" (42).

On ne peut mieux confirmer Stirner dans son opinion : cette priorité donnée à l'individu générique prouve justement que "l'émancipation complète" est une complète transposition, et que l'anthropologie reprend simplement à son compte l'asservissement de droit de l'individu à l'universel. Feuerbach aura beau rétorquer que Stirner, en prenant pour principe "le moi fini", montre qu'il méconnaît ce que signifiait

(40) Manifestes. p. 224.

(41) Ibid. p. 226.

(42) Ibid. p. 227. 
en réalité "l'essence divine" ou plutôt qu'il l'interprète encore chrétiennement. Mais Stirner se défend de rien interpréter. Comme toute forme du sacré, "l'essence divine" est simplement à détruire: quelle nécessité d'en restituer le vrai sens? En lui faisant l'honneur d'un déchiffrement, on ne fait que "contraindre le Dieu céleste à nous rejoindre avec armes et bagages"... Ajoutons qu'on évite la vraie, la seule question subversive, celle que formulera Nietzsche en présentant le Retour éternel (dans les textes que les éditeurs ont placés à la fin de la Volonté de Puissance) : à quelles conditions puis-je être assuré de ne plus penser l'infinité théologique, sous quelque forme que ce soit? quand puis-je être assuré de n'avoir pas rebaptisé le divin? Comme le dit Stirner: "Que je prenne l'espéce ou l'humanité comme modèle idéal, ou Dieu et le Christ, comment voir là une différence essentielle?.. Nos athées sont des gens pieux' ${ }^{(43)}$. N'est-il pas pieux, en effet, le langage dont use Feuerbach pour blâmer la superbe de l'Unique? "Nous nous sentons limités et imparfaits... Où donc nous délivrer de ce sentiment de limitation, sinon dans la pensée de l'espèce illimitée...?" (44). - Comment oublier mon imperfection? Comment faire de mon négatif un mauvais rêve? Le fait même de poser sans fard ces questions pieuses montre combien la religion est le texte "princeps" de l' "anthropologie" aussi bien que de la spéculation. - Non, dans l'Allemagne des années 1840, la critique de la religion n'était nullement "faite en substance". Et encore moins entamée la critique des présupposés de Hegel. - Ici commencerait l'histoire de la véritable idéologie allemande: celle que circonscrit Stirner, - le reste étant querelle de sectes.

Substitution du "genre humain" à l'infini théologique, neutralisation de l'individu par l'universel: ces seuls traits feraient déjà de l'Essence du Christianisme un lieu de formation remarquable de ce que Nietzsche détestera et dénoncera sous le nom d'“idées modernes", - entendons : produits de remplacement du christianisme. Car c'est bien de cela qu'il s'agit (et depuis la lettre de 1828 à Hegel) : remplacer la religion. D'où vient donc l'urgence de cette tâche? Feuerbach n'en fait pas mystère, - et ses phrases sont saisissantes, tant elles justifient l'analyse de Nietzsche.

(43) Stimer. Unique. p. 232, 234.

(44) Manifestes. p. 230. 
"Si l'on ne remplace pas la divinité par l'espèce, on laisse dans l'individu un vide qui, nécessairement, se comblera de nouveau par la représentation d'un Dieu, essence personnifiée de l'espèce. Seule l'espèce est capable à la fois de supprimer et de remplacer la divinité et la religion" (45).

On le voit: l'Essence du Christianisme n'est pas dédiée aux "nihilistes forts". Dieu ou l'espèce, à la limite, il importe peu, pourvu qu'on évite la condition la plus intolérable : "le vide", - le plus intolérable aussi des supplices: un besoin d'idéal dont on sait qu'il ne sera jamais satisfait. Ce que Nietzsche appellera "nihilisme", et dont le livre de Feuerbach est l'antidote, le retardateur. Et c'était peut-être un mérite suffisant pour s'attirer la reconnaissance des contemporains .

"C'est alors que parut l'Essence du Christianisme de Feuerbach. D'un seul coup, il réduisit en poussière la contradiction en replaçant sans détours le matérialisme sur le trône... Il faut avoir éprouvé soi-même l'action libératrice de ce livre pour s'en faire une idée. L'enthousiasme fut général; nous fûmes tous momentanément des "feuerbachiens". On peut voir, en lisant la Sainte Famille, avec quel enthousiasme Marx salua la nouvelle façon de voir et à quel point - malgré toutes ses réserves critiques - il fut influencé par elle" (Engels) .

(45) Ibid., iclem. 